\title{
Type Gultures and Proposed Neotype Gultures of Some Species in the Genus Bacillus
}

\author{
BY N. R. SMITH \\ 322 S. Washington Drive, Sarasota, Florida, U.S.A., \\ T. GIBSON \\ School of Agriculture, Edinburgh, \\ RUTH E. GORDON \\ Institute of Microbiology, Rutgers, The State University, \\ New Brunswick, New Jersey, U.S.A., \\ AND P. H. A. SNEATH \\ National Institute for Medical Research, Mill Hill, London, N.W. 7 \\ (Received 11 July 1963) \\ SUMMARY \\ Type cultures or proposed neotype cultures of 20 species in the genus \\ Bacillus are listed, together with their histories and their accession numbers \\ in the American Type Culture Collection, the National Collection of \\ Industrial Bacteria and the National Collection of Type Cultures.
}

\section{INTRODUCTION}

The concepts of the type species and the type culture adopted by the International Code of Nomenclature of Bacteria and Viruses (1958) are exceedingly important in microbial taxonomy. If these concepts are to be followed in classification and identification, strains typifying the established species must be available for comparison with newly isolated strains and with strains used in new tests, observations and processes. In the present paper, cultures of 20 species in the genus Bacillus are arranged in two lists. The first is a list of type cultures. The second shows cultures which we now propose as being suitable for acceptance as neotype cultures. The lists are presented in the hope that they will assist future work; no attempt is made to include all the species of Bacillus which might deserve recognition.

The strains of the first list are those designated by the author of the species as the type culture, one designated as a neotype culture by international agreement (the Marburg strain of Bacillus subtilis), or, lacking such designations, those made available to other microbiologists by the author as representing his species. When more than one strain was distributed by the author, the first dispensed or the first of a numerical or alphabetical series is regarded as the type culture. The second list contains the proposed neotype cultures for the species whose type culture has, to the best of our knowledge, been lost. The strains selected are considered suitable representatives of their respective species; they have been available for a relatively long time from a type culture collection or the collection of an investigator interested 
in the genus; and several are from the collection of the author of an accepted emendation of the species description.

The strains listed here are available from: the American Type Culture Collection, 2112 M Street, N.W., Washington 7, D.C., U.S.A. (ATCC); the National Collection of Industrial Bacteria, Torry Research Station, Aberdeen, Scotland (NCIB); the National Collection of Type Cultures, Central Public Health Laboratory, Colindale Avenue, London, N.W. 9 (NCTC). The accession number of each strain in each of the three culture collections is given. In addition, its history is shown in parentheses by the names of the persons or collections responsible for propagating and transmitting it, starting with the final source of the culture and ending with the original. Pertinent references to the species are given by Smith \& Gordon (1957) (referred to in the lists as Manual) and are not repeated here.

\section{LIST OF TYPE CULTURES}

Bacillus badius Batchelor emend. Saghafi \& Appleman. Species description: Manual, p. 623. Type culture: ATcc 14574, NCIB 9364, NCTC 10333 (N. R. Smith 663; Henry 110; Batchelor).

Bacillus coagulans Hammer. Species description: Manual, p. 622. Type culture: ATCC 7050, NCIB 9365, NCTC 10334 (N. R. Smith 609; Porter; Hammer).

Bacillus firmus Werner. Species description: Manual, p. 624. Type culture: ATCC 14575, NCIB 9366, NCTC 10335 (N. R. Smith 613; Porter; Bredemann, original strain; Werner).

Bacillus laterosporus Laubach. Species description: Manual, p. 629. Type culture: ATCC 64, NсIв 9367, NCTC 6357 (American Museum of Natural History 797; Ford 6; Laubach).

Bacillus lentus Gibson. Species description: Manual, p. 624. Type culture: ATCC 10840, NCIB 8773, NCTC 4824 (Gibson 165).

Bacillus macerans Schardinger. Species description: Manual, p. 626. Type culture: atcc 8244, NCIB 9368, NCTc 6355 (N. R. Smith 888; Porter; Pribram; Schardinger).

Bacillus pantothenticus Proom \& Knight. Species description: Manual, p. 632. Type culture: atcc 14576, NCIB 8775, NCTC 8162 (Proom CN 3028).

Bacillus pumilus Gottheil. Species description: Manual, p. 622. Type culture: ATCC 7061, NCIB 9369, NCTC 10337 (N. R. Smith 272; Löhnis; Král; Gottheil).

Bacillus sphaericus Neide. Species description: Manual, p. 633. Type culture: AтCC 14577, мсів 9370, мстс 10338 (Gibson 1013; Král, Marburg strain; Wund; Neide).

Bacillus stearothermophilus Donk. Species description: Manual, p. 627. Type culture: ATCC 12980, NCIB 8923, NCTC 10339 (N. R. Smith T 18; National Canners Association 26; Donk).

Bacillus subtilis Cohn emend. Prazmowski. Species description: Manual, p. 620. Type culture: ATCC 6051, NCIB 3610, NCTC 3610 (Conn, Marburg strain).

\section{LIST OF PROPOSED NEOTYPE CULTURES}

Bacillus alvei Cheshire \& Cheyne. Species description: Manual, p. 626. Proposed neotype culture: ATCC 6344, NCIB 9371, NCTc 6352 (N. R. Smith 662; Lochhead 
127). The original strains studied by Cheshire and Cheyne have not been found and may be presumed to have been lost. Lochhead isolated his strain 127 from a honeycomb with European foulbrood in 1925 and distributed it as a representative of the species. Because it is typical and has been available for some time, it is proposed as a neotype culture.

Bacillus anthracis Cohn emend. Koch. Species description: Manual, p. 618. Proposed neotype culture: ATCC 14578, NCIB 9388, NCTC 10340 (Microbiological Research Establishment, Porton, Wiltshire, England; Vollum; Dunkin). The anthrax bacillus (together with Bacillus mycoides and some insect pathogens such as Bacillus thuringiensis) overlaps with Bacillus cereus in many features. It is treated here as a separate species from the nomenclatural standpoint, without implying that we do or do not regard it taxonomically as a separate species. The original strains of Koch appear to have been lost in 1945, and none can now be traced. This strain, the Vollum strain, was isolated from bovine anthrax, has retained its virulence, and is typical culturally; it has been widely studied and is proposed as the neotype culture.

Bacillus brevis Migula emend. Ford. Species description: Manual, p. 630. Proposed neotype culture: ATCC 8246, NCIB 9372, NCTC 2611 (N. R. Smith 604; Porter; NCTC; Ford 27 B). The first definitive characterization of this species was provided by Ford. His strain $27 \mathrm{~B}$, which he deposited in culture collections, is believed to be the oldest available as a neotype of the species.

Bacillus cereus Frankland \& Frankland. Species description: Manual, p. 617. Proposed neotype culture: ATCC 14579, NCIB 9373, NCTC 2599 (Gibson 971; NCTC; Ford 13). There appears to be no evidence that the original culture of $B$. cereus was preserved. An important and comparatively early contribution concerning this species was made by Lawrence \& Ford (1916). Strain 13 which Ford sent to culture collections is still typical and is now proposed for acceptance as the neotype of the species.

Bacillus circulans Jordan emend. Ford. Species description: Manual, p. 628. Proposed neotype culture: ATCC 4513, NCIB 9374, NCTC 2610 (Ford 26). This species was adequately characterized for the first time by Ford. His strain 26 which he supplied to culture collections is therefore proposed as the neotype culture.

Bacillus licheniformis (Weigmann) Chester emend. Gibson. Species description: Manual, p. 619. Proposed neotype culture: ATCC 14580, NCIB 9375, NCTC 10341 (Gibson 46). No record has been found to indicate that Weigmann's original culture of this species was preserved. Gibson, who provided an amplified characterization of the species, distributed his strain $\mathbf{4 6}$ to culture collections, and it is proposed as the neotype of the species.

Bacillus megaterium De Bary. Species description: Manual, p. 616. Proposed neotype culture: ATCC 14581, NCIB 9376, NCTC 10342 (Gibson 1060; NCTC; Ford 19). De Bary's culture of this species does not appear to have been available to subsequent workers and may be presumed to have been lost. In view of the important contribution to the definition of the species made by Lawrence \& Ford (1916), the strain that Ford supplied to culture collections (his strain 19) is proposed as the neotype.

Bacillus pasteurii (Miquel) Migula. Species description: Manual, p. 633. Proposed neotype culture: ATCC 11859, NCIB 8841, NCTC 4822 (Gibson 22). There appears 
to be no reference in subsequent literature to the original culture of this species, thus indicating that Miquel did not propagate a specimen. The strain proposed as a neotype has been maintained in culture collections for a considerable time and, unlike some older cultures of the species, it has retained its original properties.

Bacillus polymyxa (Prazmowski) Migula. Species description: Manual, p. 625. Proposed neotype culture: ATcc 842, NсIB 8158, nctc 10343 (Kluyver). Prazmowski's original culture of this species has not been mentioned by later writers, and there is a virtual certainty that it no longer exists. The proposed neotype culture was chosen for the reason that it has been a subject of investigation over a long period at Delft and elsewhere.

This study was supported in part by research grant G-5424 from the National Science Foundation, Washington, D.C., U.S.A.

\section{REFERENCES}

International Code of Nomenclature of Bacteria and Viruses (1958), p. 17. Ed. The Editorial Board of the International Committee on Bacteriological Nomenclature. Ames: Iowa State University Press.

LAwRENCE, J. S. \& FORD, W. W. (1916). Studies on aerobic spore-bearing non-pathogenic bacteria. J. Bact. 1, 284.

Smith, N. R. \& Gordon, R. E. (1957). In Bergey's Manual of Determinative Bacteriology, 7th ed. Baltimore: The Williams and Wilkins Company. 